\title{
Holey topological thermoelectrics
}

\author{
O. A. Tretiakov, Ar. Abanov, and Jairo Sinova \\ Department of Physics, Texas A\&̈M University, College Station, TX 77843, USA
}

(Dated: June 30, 2011)

\begin{abstract}
We study the thermoelectric properties of three-dimensional topological insulators with many holes (or pores) in the bulk. We show that at high density of these holes the thermoelectric figure of merit $Z T$ can be large due to the contribution of the conducting surfaces and the suppressed phonon thermal conductivity. The maximum efficiency can be tuned by an induced gap in the surface states dispersion through tunneling or external magnetic fields. The large values of $Z T$, much higher than unity for reasonable parameters, make this system a strong candidate for applications in heat management of nanodevices, especially at low temperatures.
\end{abstract}

Efficient heat management in nanodevices and energy conversion from wasted heat have been some of the key driving motivations for the search of new thermoelectric materials [1 14]. Their efficiency rests on the ability of a material to have a low thermal conductivity and high thermopower and electric conductivity. The large thermopower requires steep dependence of the electronic density of states on energy, which can be achieved by having the chemical potential close to the bottom of a band. The relatively high conductivity demands the gap to be low. These combined requirements point to semiconductors with heavy elements as the best candidates for the thermoelectric materials.

A typical band gap for an efficient thermoelectric device at room temperature should be of the order of few hundred meV. Such a low gap can appear in the materials either accidentally or due to a large enough spinorbit coupling that leads to the inversion of the band structure, thus providing a band-gap of the order of the spin-orbit coupling energy. This latter class of materials, besides being highlighted by their high thermoelectric efficiency, has been identified as having an insulating bulk and topologically protected conducting surfaces with Dirac-like band structure [15-18; ; hence their name, topological insulators (TI).

The bulk thermoelectric properties of this family of semiconductors have been extensively studied both theoretically $[6] 8$ and experimentally $[10-13]$. The rather high thermoelectric efficiency found in these materials is the property of the band structure and the heavy atomic masses, and is unrelated to the contribution of the protected surface states - the main focus of this paper. As with most thermoelectric materials, the main stumbling block for increasing the thermoelectric efficiency is the high phonon thermal conductivity. There have been many attempts to decrease it while leaving the electric conductivity intact [19, 20], e.g., by introducing disorder in the material that suppresses phonon transport (phononic glass). The bulk electronic states are however often affected by this disorder as well and it is very difficult to reduce thermal conductivity without also reducing thermopower and electric conductivity [1].

Here we propose a new approach to increase thermoelectric efficiency by combining the novel properties of the TI materials, i.e., their protected surface states,

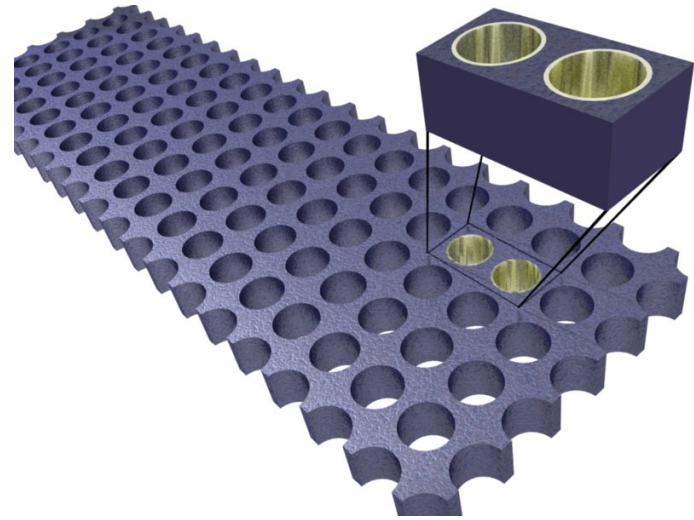

FIG. 1. A sample made of a holey 3D topological insulator. These holes increase the surface to bulk ratio and reduce its phonon thermal conductivity. The inset shows two such holes with the Dirac like metallic surface states.

with methods to reduce thermal conductivity that can enhance the contribution to the conductivity and thermopower from these topological surface states by increasing the surface to volume ratio.

The thermopower efficiency is characterized by the dimensionless thermoelectric figure of merit $Z T=$ $\sigma S^{2} T / \kappa$, where $\sigma, S, \kappa$, and $T$ are the electric conductivity, thermopower, thermal conductivity, and temperature, respectively. This quantity corresponds to the ratio of the output power to the rate of the heat power consumption under an applied temperature gradient.

The high surface to bulk ratio can be achieved by nanostructuring 3D topological insulators with many holes in the transport direction. The surface of each hole behaves as a 2D conductor. The effect of the holes is threefold: 1) to suppress phonon thermal conductivity the strongest limiting factor for $Z T, 2)$ to increase conductivity due to a high contribution of conducting surface, and 3) to allow for the surface states to tunnel and create a smaller subgap to increase $Z T$ further. A sketch of the sample made of a 3D topological insulator with a high density of holes is depicted in Fig. 1. Even at high density of these holes, the topologically protected surface states remain robust. It has been shown that these states survive in the films as thin as of three or more quintuple 
layers [21].

Reducing thermal conductivity caused by the increased phonon scattering off grain boundaries and defects has been experimentally confirmed recently. For example, in BiSbTe bulk alloys higher $Z T$ has been observed in experiments with nanocrystalline bulk samples made by pressing nanopowders at high temperatures [19. Furthermore, high density of periodic nanoscale holes in silicon thin films has been shown to reduce the thermal conductivity by two orders of magnitude [20.

The unique feature of the topological insulators is that, although they have a bulk energy gap of $2 \Delta_{0}$, they also have ungaped surface states. These propagating states are confined to the close proximity of the surfaces. Their existence is protected by the topology of the bulk band structure. These states have cone-like 2D Dirac spectrum, $E= \pm v \hbar|k|$, where $v$ is the constant Dirac electron velocity [22]. Application of a magnetic field or hybridization of these states due to close proximity of two surfaces can induced a controllable Dirac subgap $\Delta[23,24$. Then the spectrum takes the form $E= \pm \sqrt{v^{2} \hbar^{2} k^{2}+\Delta^{2}}$. Generally this subgap is much smaller than the bulk gap, $\Delta \ll \Delta_{0}$. For $3 \mathrm{D}$ TIs $2 \Delta_{0}$ is roughly from $0.15 \mathrm{eV}$ (for $\mathrm{Bi}_{2} \mathrm{Te}_{3}$ ) to $0.3 \mathrm{eV}$ (for $\mathrm{Bi}_{2} \mathrm{Se}_{3}$ ).

To study the transport properties of these materials we use linear response theory [25, 26]. The electric $\left(j^{e}\right)$ and thermal $\left(j^{q}\right)$ currents are given by linear combinations of the chemical potential and temperature gradients: $j^{e} / e=L_{0} \nabla \mu+L_{1}(\nabla T) / T$ and $j^{q}=-L_{1} \nabla \mu-L_{2}(\nabla T) / T$, where $e$ is the electron charge. Using Onsager relations, one can find from these equations the electrical conductivity $\sigma=e^{2} L_{0}$, thermopower $S=-L_{1} /\left(e T L_{0}\right)$, and electronic thermal conductivity $\kappa_{e}=\left(L_{0} L_{2}-L_{1}^{2}\right) /\left(T L_{0}\right)$. The figure of merit, $Z T$, can then be represented in terms of these linear coefficients as

$$
Z T=\frac{L_{1}^{2}}{L_{0}\left(L_{2}+\kappa_{p h} T\right)-L_{1}^{2}} .
$$

Here $\kappa_{p h}$ is the phonon contribution to the thermal conductivity in the bulk (phonon contribution to the thermal conductivity for the surface TI states is much smaller than that in the bulk). In Eq. (1) it is assumed that the transport coefficients have bulk and surface contributions $L_{n}=L_{b, n}+L_{s, n} / \mathcal{D}$, where $\mathcal{D}=\left(A-\sum_{n} \pi R_{n}^{2}\right) / \sum_{n} 2 \pi R_{n}$ is the factor related to surface/bulk ratio (porosity) and has dimension of length. The parameter $\mathcal{D}$ characterizes the average distance between the pores (holes) of the average radius $R$, see Fig. 2 (b).

It follows from Eq. (1) that at small $\mathcal{D}$, the contribution to $Z T$ mostly comes from $2 \mathrm{D}$ surface states and in this limit $Z T$ is given by

$$
Z T_{2 D}=\frac{L_{s, 1}^{2}}{L_{s, 0} L_{s, 2}-L_{s, 1}^{2}}
$$

$Z T_{2 D}$ is shown in the inset of Fig. 3 for $\Delta /\left(k_{B} T\right)=3$ as a function of the chemical potential. The color plot of (a)

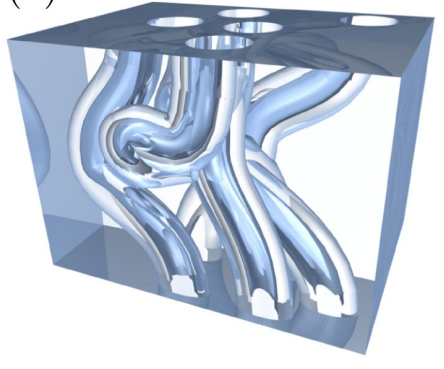

(b)

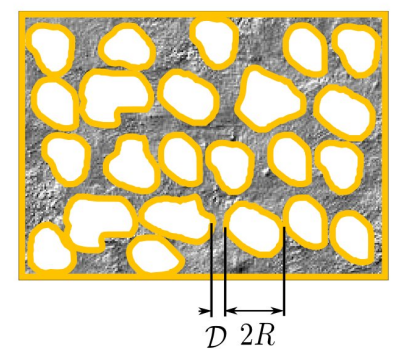

FIG. 2. (a) A sample of 3D topological insulator with randomly distributed pores. (b) Top view of small part of the sample with the holes. $\mathcal{D}$ represents the average distance between randomly distributed pores (or holes).

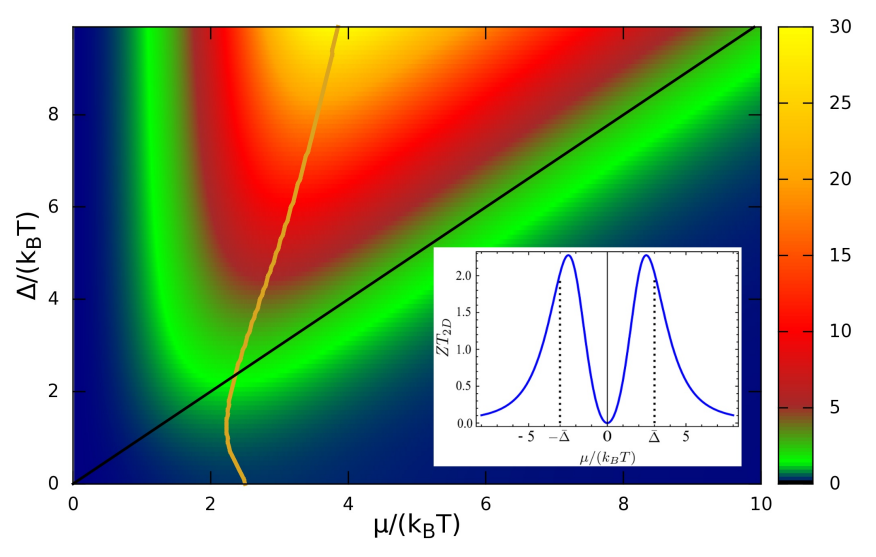

FIG. 3. The color plot of $Z T$ only for the surface states as a function of the induced subgap $\Delta /\left(k_{B} T\right)$ and chemical potential $\mu /\left(k_{B} T\right)$ measured in units of temperature. The inset shows $Z T$ calculated only for the surface states with the subgap $\Delta /\left(k_{B} T\right)=3$. The solid golden line represents the maximum $Z T$ at a fixed $\Delta$. The black line corresponds to $\mu_{\max }=\Delta$.

$Z T_{2 D}$ as a function of both the induced subgap $\Delta /\left(k_{B} T\right)$ and chemical potential $\mu /\left(k_{B} T\right)$ is shown in Fig. 3 . For a fixed induced gap $\Delta /\left(k_{B} T\right)$ we plot the location of the maximum $Z T$ achievable by tuning the chemical potential (golden line in Fig. 3). The very high values of $Z T$ in reality are not reachable since any small contribution from the phonon thermal conductivity will reduce $Z T$.

The phononic contribution to the thermal conductivity for the holey sample can be characterized by the dimensionless parameter $K_{p h}=2 \kappa_{p h} \mathcal{D} h^{2} /\left(\tau k_{B}^{3} T^{2}\right)$. Here the phonon thermal conductivity can be estimated to be $\kappa_{p h} \approx 1 \mathrm{Wm}^{-1} \mathrm{~K}^{-1}$ (as for $\mathrm{Bi}_{2} \mathrm{Te}_{3}$ ) and the average distance between the holes reaching $\mathcal{D} \sim 10 \mathrm{~nm}$. Taking the relaxation time $\tau \approx 10^{-11} \mathrm{~s}$, at room temperatures we estimate $K_{p h} \sim 1$ for these rather conservative values of parameters. In Fig. 4 we show $Z T$ as a function of the induced subgap $\Delta /\left(k_{B} T\right)$ and chemical potential $\mu /\left(k_{B} T\right)$ for the phononic bulk contribution characterized by the dimensionless parameter $K_{p h}=3$. Although reduced considerably from its pure $2 \mathrm{D}$ value, $Z T$ remains sub- 


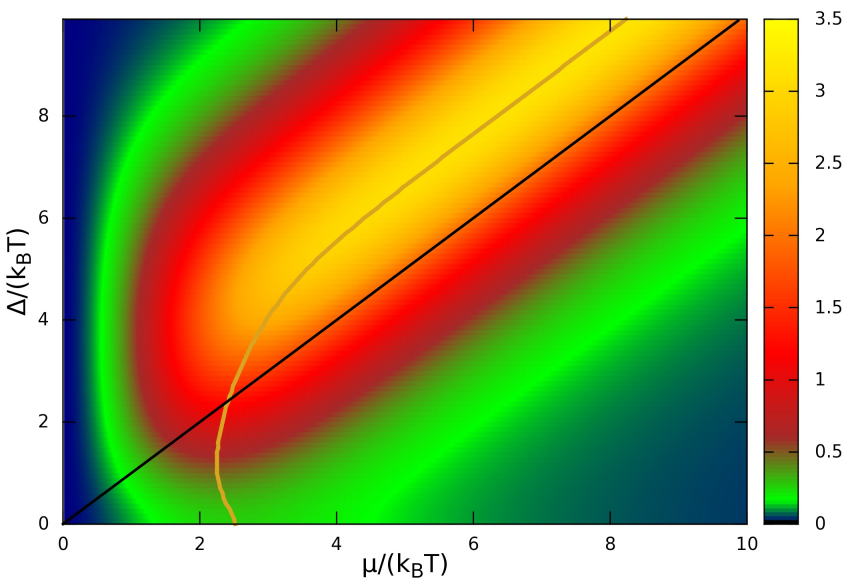

FIG. 4. $Z T$ as a function of the induced subgap $\Delta /\left(k_{B} T\right)$ and chemical potential $\mu /\left(k_{B} T\right)$ with dimensionless parameter $K_{p h}=3$ characterizing the phonon contribution to the thermal conductivity from the bulk states. The solid orange line represents the maximum $Z T$ at a fixed $\Delta$.

stantially larger than any value so far achieved in these materials and can be tuned significantly by changing the chemical potential, e. g., by gating the system.

We also investigate the relation between the induced subgap $\Delta /\left(k_{B} T\right)$ and the chemical potential $\mu_{\max } /\left(k_{B} T\right)$ to obtain the maximum value of $Z T$ for different phononic bulk contributions. This result is shown in Fig. 5. For small phonon thermal conductivity (small $\left.K_{p h}\right)$ the $Z T$ is maximal when the chemical potential is placed within the subgap, $\mu<\Delta$. In the limit of large $K_{p h}$ the maximum $Z T$ is reached when the chemical potential and the gap match. This can be seen in Fig. 5 , where the line corresponding to $K_{p h}=90$ almost coincides with the dashed line $\Delta=\mu_{\max }$ for rather large $\mu_{\max } /\left(k_{B} T\right)$.

Finally, we point out that the large values of $Z T$ obtained in the absence of phonon thermal conductivity shown in Fig. 3 is not the entire story. As at large $\Delta /\left(k_{B} T\right)$ and $\mu /\left(k_{B} T\right)$ these thermoelectrics are indeed very efficient but not effective - the power factor $\sigma S^{2}$, describing how much power one can produce, is small in this limit. This is shown in Fig. 6 as a plot of the maximal $Z T$ as a function of the power factor. The dimensionless power output $L_{1}^{2} / L_{0} \propto \sigma S^{2}$ as a function of the induced subgap $\Delta /\left(k_{B} T\right)$ and chemical potential $\mu /\left(k_{B} T\right)$ is shown in the inset of Fig. 6.

Within these materials, there are extensive competing mechanisms that contribute to the thermal transport and thermoelectric efficiency. In order to distinguish that a particular increase of $Z T$ is associated with the topological protected states, we propose a simple measurement of the transport coefficients with and without a magnetic field applied, parallel to the transport direction, to increase the induced gap of the surface states in the energy spectrum. The effect of the magnetic field will be more pronounced when the temperature is smaller than the in-

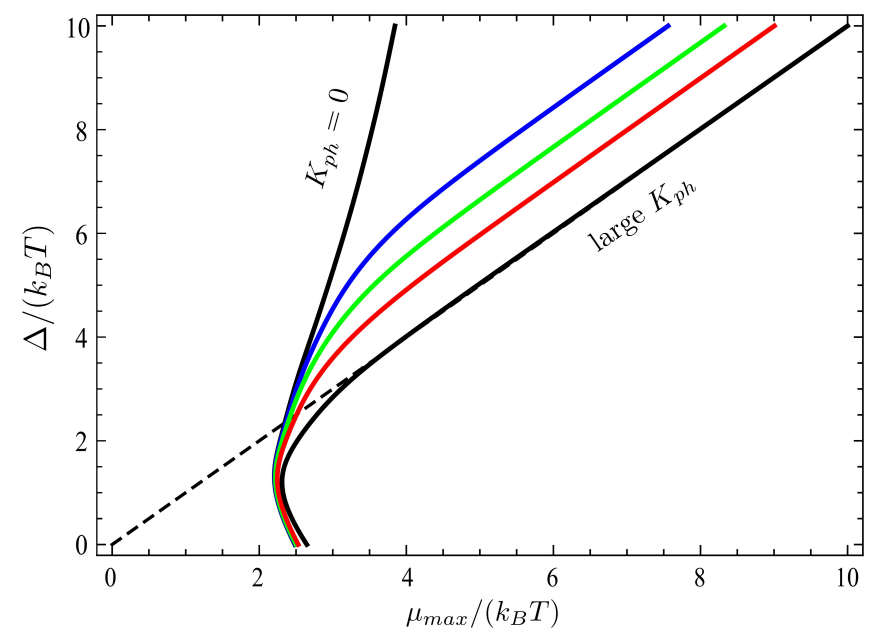

FIG. 5. Induced subgap $\Delta /\left(k_{B} T\right)$ as a function of chemical potential $\mu_{\max } /\left(k_{B} T\right)$ for the maximum value of $Z T$. The dimensionless parameter $K_{p h}$ characterizing the contribution of the phonon thermal conductivity is taken to be $0 ; 1 ; 3 ; 9$; and 90 . The dashed line $\mu_{\max }=\Delta$ is shown for comparison.

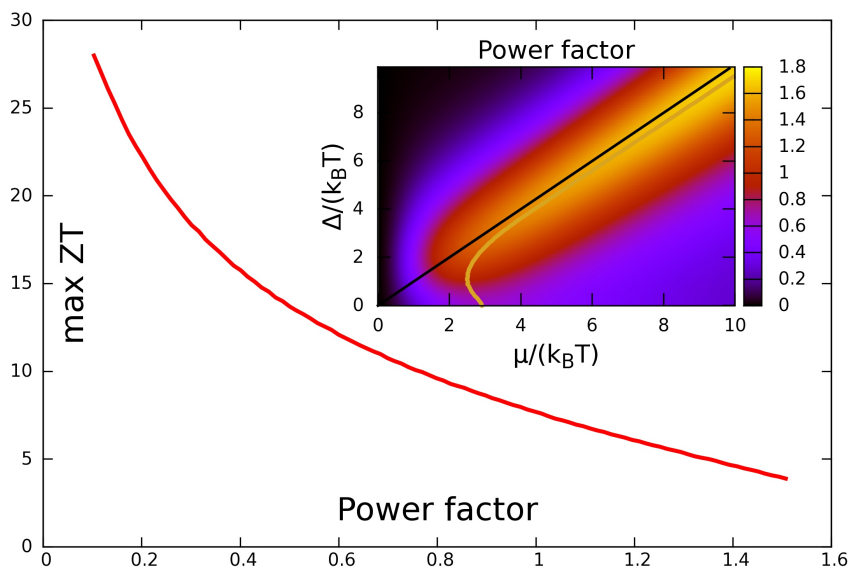

FIG. 6. Maximum $Z T$ as a function of the dimensionless power factor, $L_{1}^{2} / L_{0} \propto \sigma S^{2}$. The inset shows the power factor as a function of the induced gap $\Delta /\left(k_{B} T\right)$ and chemical potential $\mu /\left(k_{B} T\right)$ measured in units of temperature.

duced gap. For fields of $\sim 10$ Tesla a gap of the order of several meV is expected in materials such as $\mathrm{Bi}_{2} \mathrm{Se}_{3}$ [27, 28].

It is important to note that our consideration is not limited to periodic holey structures. It is also applicable to porous materials with random size and location of the holes (pores) as shown in Fig. 2(a). In this case some of the surface states can be situated too close to each other so that the back-scattering in them will be increased, meanwhile the phonon part of thermal conductivity will be reduced due to stronger trapping of phonons in chaotic structures. Therefore, $Z T$ in porous materials can be comparable to those with the periodically placed holes. 


\section{METHODS}

To estimate the surface contribution of TI to the transport coefficients $L_{n}$ we assume the bands to be Dirac-like with a subgap $\Delta$ and use Boltzmann equation in the relaxation time approximation,

$$
L_{s, n}=-2 \sum_{i} \int_{-\infty}^{\infty} \tau\left(\frac{\partial E_{i}}{\partial \hbar k}\right)^{2} f^{\prime}\left(E_{i}\right)\left(E_{i}-\mu\right)^{n} \frac{d^{2} k}{4 \pi^{2}}
$$

Here the sum is over the upper and lower bands, $i= \pm 1$, and $f^{\prime}(E)=\partial f / \partial E$ with $f=1 /\left(e^{(E-\mu) /\left(k_{B} T\right)}+1\right)$ being the Fermi distribution function. Then taking relaxation time $\tau$ to be independent of energy, we find

$L_{s, n}=\frac{\tau\left(k_{B} T\right)^{1+n}}{2 h^{2}} \int_{\bar{\Delta}}^{\infty} d x \frac{x^{2}-\bar{\Delta}^{2}}{x}\left[\frac{(x-\bar{\mu})^{n}}{\cosh ^{2} \frac{x-\bar{\mu}}{2}}+\frac{(-x-\bar{\mu})^{n}}{\cosh ^{2} \frac{x+\bar{\mu}}{2}}\right] \begin{aligned} & \text { This expression was used to obtain the results shown in } 4 \text { and } 5 . \\ & \text { Figs. }\end{aligned}$

where $h$ is Planck constant, $\bar{\Delta}=\Delta /\left(k_{B} T\right)$, and $\bar{\mu}=$ $\mu /\left(k_{B} T\right)$.

To estimate the bulk contribution to $L_{n}$ we assume the bands to be parabolic and find in the relaxation time approximation for the conduction band,

$$
L_{b, n}=-\tau \int_{\Delta_{0}}^{\infty} D(E)\left(\frac{\partial E}{\partial \hbar k}\right)^{2} f^{\prime}(E)(E-\mu)^{n} d E
$$

where $D(E)$ is the density of states. When the chemical potential is far below the bottom of the conduction band, $\left(\Delta_{0}-\mu\right) /\left(k_{B} T\right) \gg 1$, the contribution from the bulk to the transport coefficients is exponentially suppressed, $L_{b, n} \propto e^{-\left(\Delta_{0}-\mu\right) /\left(k_{B} T\right)}$, and can be safely neglected [29]. The same applies for the valence band contribution when the chemical potential is far away from the band edge.

Then the thermoelectric transport is dominated by the surface states and the only sensible contribution from the bulk is to the phonon thermal conductivity $\kappa_{p h}$, so that the figure of merit takes the form

$$
Z T=\frac{L_{s, 1}^{2}}{L_{s, 0}\left(L_{s, 2}+\mathcal{D} \kappa_{p h} T\right)-L_{s, 1}^{2}} .
$$

\section{ACKNOWLEDGMENTS}

We thank M. Bakker, J. Heremans, C. Jaworski, J. E. Moore, O. Mryasov, and D. Pesin for insightful discussions. This work was supported by NSF under Grant No. DMR-0547875, Grant No. 0757992, NSF-MRSEC DMR-0820414, ONR-N000141110780, SWAN, and by the Welch Foundation (A-1678).
[1] G. J. Snyder and E. S. Toberer, Nat. Mater., 7, 105 (2008)

[2] T. M. Tritt, Science, 283, 804 (1999).

[3] L. D. Hicks and M. S. Dresselhaus, Phys. Rev. B, 47, 12727 (1993)

[4] S. Mukerjee and J. E. Moore, Appl. Phys. Lett., 90, 112107 (2007)

[5] T. Markussen, A.-P. Jauho, and M. Brandbyge, Phys. Rev. Lett., 103, 055502 (2009).

[6] R. Takahashi and S. Murakami, Phys. Rev. B, 81, 161302 (2010)

[7] P. Ghaemi, R. S. K. Mong, and J. E. Moore, Phys. Rev. Lett., 105, 166603 (2010)

[8] O. A. Tretiakov, A. Abanov, S. Murakami, and J. Sinova, Appl. Phys. Lett., 97, 073108 (2010)

[9] H.-K. Lyeo, A. A. Khajetoorians, L. Shi, K. P. Pipe, R. J. Ram, A. Shakouri, and C. K. Shih, Science, 303, 816 (2004)

[10] R. Venkatasubramanian, E. Siivola, T. Colpitts, and B. O'Quinn, Nature, 413, 597 (2001)

[11] Y. Zhang, C. L. Hapenciuc, E. E. Castillo, T. BorcaTasciuc, R. J. Mehta, C. Karthik, and G. Ramanath, Appl. Phys. Lett., 96, 062107 (2010)

[12] D. Teweldebrhan, V. Goyal, M. Rahman, and A. A. Balandin, Appl. Phys. Lett., 96, 053107 (2010)

[13] D. Teweldebrhan, V. Goyal, and A. A. Balandin, Nano Lett., 10, 1209 (2010)
[14] Y. Dubi and M. Di Ventra, Rev. Mod. Phys., 83, 131 (2011).

[15] X.-L. Qi and S.-C. Zhang, Physics Today, 63, 33 (2010).

[16] L. Fu, C. L. Kane, and E. J. Mele, Phys. Rev. Lett., 98, 106803 (2007)

[17] D. Hsieh, D. Qian, L. Wray, Y. Xia, Y. S. Hor, R. J. Cava, and M. Z. Hasan, Nature, 452, 970 (2008).

[18] Y. L. Chen, J. G. Analytis, J.-H. Chu, Z. K. Liu, S.-K. Mo, X. L. Qi, H. J. Zhang, D. H. Lu, X. Dai, Z. Fang, S. C. Zhang, I. R. Fisher, Z. Hussain, and Z.-X. Shen, Science, 325, 178 (2009)

[19] B. Poudel, Q. Hao, Y. Ma, Y. Lan, A. Minnich, B. Yu, X. Yan, D. Wang, A. Muto, D. Vashaee, X. Chen, J. Liu, M. S. Dresselhaus, G. Chen, and Z. Ren, Science, 320, 634 (2008)

[20] J. Tang, H.-T. Wang, D. H. Lee, M. Fardy, Z. Huo, T. P. Russell, and P. Yang, Nano Lett., 10, 4279 (2010).

[21] K. Park, J. J. Heremans, V. W. Scarola, and D. Minic, Phys. Rev. Lett., 105, 186801 (2010).

[22] We consider $v$ to be energy independent in a wide range of energies.

[23] J. Linder, T. Yokoyama, and A. Sudbø, Phys. Rev. B, 80, 205401 (2009).

[24] H.-Z. Lu, W.-Y. Shan, W. Yao, Q. Niu, and S.-Q. Shen, Phys. Rev. B, 81, 115407 (2010)

[25] N. W. Ashcroft and N. D. Mermin, Solid State Physics (Sauders College Publishing, Fort Worth, 1976). 
[26] M. P. Marder, Condensed Matter Physics (John Wiley \& Sons, Inc., New York, 1976).

[27] A. A. Zyuzin and A. A. Burkov, Phys. Rev. B, 83, 195413 (2011)

[28] J. G. Analytis, R. D. McDonald, S. C. Riggs, J. Chu, G. S. Boebinger, and I. R. Fisher, Nat. Phys., 6, 960
$(2010)$

[29] We note that taking into account the fact that the extended and localized states in the bulk are separated by the mobility edge $E_{m}$ which is measured from the bottom of the band changes $\Delta_{0}$ to $\Delta_{0}-E_{m}$ in these estimates and only makes the inequalities to be even stronger. 